\title{
SCALE AND TIDY SUBGROUPS FOR WEYL-TRANSITIVE AUTOMORPHISM GROUPS OF BUILDINGS
}

\author{
U. BAUMGARTNER, J. PARKINSON, AND J. RAMAGGE
}

\begin{abstract}
We consider closed, Weyl-transitive groups of automorphisms of thick buildings. For each element of such a group, we derive a combinatorial formula for its scale and establish the existence of a tidy subgroup for it that equals the stabilizer of a simplex. Simplices whose stabilizers are tidy for some element of the group are characterized in terms of the minimal set of the isometry induced by the element on the Davis-realisation of the building and in terms of the Weyl-distance between them and their image. We use our results to derive some topological properties of closed, Weyl-transitive groups of automorphisms.
\end{abstract}

\section{INTRODUCTION}

Tidy subgroups and the scale function provide important structural information on a totally disconnected locally compact group and have found diverse applications including the commensurated subgroup problem in arithmetic groups [21], and random walks and ergodic theory [11, 14. Describing tidy subgroups for a given group and computing the scale of elements is however a challenging problem. For example, see [12] for the computation of the scale function for $p$-adic Lie groups. In a more general setting the main challenges in characterising tidy subgroups and calculating the scale function lie in either finding a description of the group that is suitable for the task, or conversely finding a way to express the concepts of tidyness and scale within the given description of the group.

Every automorphism group of a locally finite cell complex is totally disconnected and locally compact in the compact-open topology. In this note we examine the example of the groups of automorphisms of a locally finite building with sufficiently transitive action. This important class of examples includes the subclasses of Lie groups over nonarchimedean local fields and Kac-Moody groups defined over finite fields. The methods used exploit the beautiful and rich geometry of the underlying building. Using this geometric approach we are able to provide a concrete description of the tidy subgroups, and a method to calculate the scale of an element directly in terms of the combinatorics of the action on the building.

Date: March 24, 2022.

2010 Mathematics Subject Classification. 22D05, 52E24, 20E36, $20 \mathrm{~F} 65$.

Key words and phrases. totally disconnected locally compact groups, scale function, tidy subgroups, buildings.

This research was supported by Australian Research Council grant DP150100060. 


\section{Tidy Subgroups And THE SCALE}

Every totally disconnected, locally compact group has compact, open subgroups by Van Dantzig's Theorem. Given a continuous automorphism, $\alpha$, of such a group with continuous inverse, and a compact, open subgroup $V$, the index $\mid \alpha(V): \alpha(V) \cap$ $V \mid$ measures the distortion of $V$ under $\alpha$. This index is finite because $\alpha(V)$ is again compact and open and all compact, open subgroups of a group are commensurable.

Since the indices $|\alpha(V): \alpha(V) \cap V|$ are integers, for every $\alpha$ there exist compact, open subgroups that minimize this index. These subgroups are called tidy for $\alpha$ and the minimal distortion index is called the scale of $\alpha$, denoted $s(\alpha)$.

In this paper we restrict our attention to inner automorphisms. A compact, open subgroup will be called tidy for a group element $g$ if and only if it is tidy for inner conjugation by $g$, given by $x \mapsto g x g^{-1}$. Likewise the scale of inner conjugation by $g$ will be denoted $s(g)$ and called the scale of $g$.

There are several ways to characterize tidy subgroups. A criterion for tidiness due to Möller will be the one we most often use in this paper.

Lemma 2.1 ([17, Corollary 3.5]). A compact, open subgroup $U$ is tidy for an element $g$ if and only if

$$
\left|U: U \cap g^{-n} U g^{n}\right|=\left|U: U \cap g^{-1} U g\right|^{n} \quad \text { for all integers } n \geq 0 .
$$

The following interpretation of tidy subgroups motivates our geometric approach to tidy subgroups.

Lemma 2.2 ([5, Lemma 2(0)]). A compact, open subgroup is tidy for $\alpha$ if and only if the displacement $\mathbf{d}(\alpha(O), O)$ with respect to the metric $\mathbf{d}$ on the set of compact, open subgroups defined by $\mathbf{d}(V, W):=\log (|V: V \cap W| \cdot|W: W \cap V|)$ is minimal at $O$. The value of this minimal displacement is $\log (s(\alpha))+\log \left(s\left(\alpha^{-1}\right)\right)$.

The following formulas for the scale will be of central importance in section 5 .

Lemma 2.3. Suppose that $O$ is tidy for $g$. Then

$$
s(g)=\left|g O g^{-1}: g O g^{-1} \cap O\right|=\left|O: O \cap g^{-1} O g\right|=|O \backslash O g O|
$$

Proof. The first formula for the scale is immediate from the definition. The second one follows by invariance of the subgroup index under conjugation. The third one can be verfied for example using Lemma 3.9 in [16] or Section 3.1 in [13]

\section{FRAMEWORK FOR BUILDINGS}

The theory of buildings grew from the fundamental work of Jacques Tits starting in the 1950s. The initial impetus was to give a uniform description of semisimple Lie groups and algebraic groups by associating a geometry to each such group. This "geometry of parabolic subgroups" later became known as the (spherical) building of the group [22]. The utility and scope of building theory has since far outgrown the original raison d'être, with crucial applications in the theory of Lie 
groups defined over nonarchimedean local fields (the affine buildings), and more broadly the theory of Kac-Moody groups. We will describe the latter connection in Example 3.1 below.

Our main reference for the theory of buildings is [1. Let us briefly fix notation. Let $(W, S)$ be a Coxeter system (with $|S|<\infty$ ) and let $\ell: W \rightarrow \mathbb{Z}_{\geq 0}$ be the length function on $W$ with respect to the generating set $S$. A building of type $(W, S)$ is a pair $(\Delta, \delta)$ where $\Delta$ is a nonempty set (whose elements are called chambers) and $\delta: \Delta \times \Delta \rightarrow W$ is a function (called the Weyl distance function) such that if $x, y \in \Delta$ then the following conditions hold, where $w=\delta(x, y)$ :

(1) $w=1$ if and only if $x=y$.

(2) For each $s \in S$ there is $z \in \Delta$ with $\delta(y, z)=s$ and $\delta(x, z)=w s$.

(3) If $z \in \Delta$ with $\delta(y, z)=s \in S$ then $\delta(x, z) \in\{w s, w\}$. Moreover, if $\ell(w s)=\ell(w)+1$ then $\delta(x, z)=w s$.

The Coxeter group $W$ is itself a building of type $(W, S)$, with Weyl distance function given by $\delta\left(w, w^{\prime}\right)=w^{-1} w^{\prime}$ (this building is called the Coxeter complex). Every subset of $\Delta$ which is $\delta$-isometric to the Coxeter complex is called an apartment of $\Delta$, and a fundamental property of buildings is that given any two chambers $x, y \in \Delta$ there exists an apartment containing them both.

A Coxeter system $(W, S)$ is called spherical if $|W|<\infty$, and a building is called spherical if its Coxeter system is spherical. In this paper we are primarily concerned with non-spherical buildings. However, as described below, certain spherical subbuildings play an important role.

If $s \in S$, chambers $x, y \in \Delta$ are $s$-adjacent (written $x \sim_{s} y$ ) if $\delta(x, y)=s$. The building $\Delta$ is:

(1) locally finite if $\left|\left\{y: x \sim_{s} y\right\}\right|$ is finite for every $x \in \Delta$ and $s \in S$,

(2) thick if $\left|\left\{y: x \sim_{s} y\right\}\right| \geq 2$ for every $x \in \Delta$ and $s \in S$,

(3) regular if it is locally finite and $q_{s}=\left|\left\{y: x \sim_{s} y\right\}\right|$ is independent of $x \in \Delta$.

Henceforth, $(\Delta, \delta)$ denotes a non-spherical locally finite thick building of type $(W, S)$. Moreover, our assumptions on the group $G$ of automorphism of $\Delta$ (see below) will imply that $(\Delta, \delta)$ is also regular. In this case, if $w \in W$ then the value

$$
q_{w}:=q_{s_{1}} \cdots q_{s_{n}}
$$

is independent of the particular reduced decomposition $w=s_{1} \cdots s_{n}$ for $w$ chosen (see, for example, [20, Proposition 2.1]).

A gallery of type $\left(s_{1}, \ldots, s_{n}\right)$ from a chamber $x$ to a chamber $y$ is a sequence $x=x_{0} \sim_{s_{1}} x_{1} \sim_{s_{2}} \cdots \sim_{s_{n}} x_{n}=y$. We say that this gallery has length $n$. A key property is that this gallery has minimal length amongst all galleries from $x$ to $y$ if and only if $\ell\left(s_{1} \cdots s_{n}\right)=n$. In other words, a gallery is minimal if and only if its type is reduced in $W$.

Example 3.1. Let $G=G(\mathbb{F})$ be Chevalley group over a field $\mathbb{F}$, with Coxeter system $(W, S)$. Let $B$ be a 'Borel subgroup' (generated by the positive root subgroups 
and the torus elements). Then $(\Delta, \delta)$ is a spherical building of type $(W, S)$, where $\Delta=G / B$ and $\delta(g B, h B)=w$ if and only if $g^{-1} h \in B w B$. Thus $(\Delta, \delta)$ may be viewed as a combinatorial/geometric object encoding the structure of the Bruhat decomposition $G=\bigsqcup_{w \in W} B w B$, which highlights the original motivation for the invention of buildings.

There is a far-reaching generalisation of this setup, where $G=G(\mathbb{F})$ is a KacMoody group over $\mathbb{F}$ with Coxeter system $(W, S)$. In this more general situation $\Delta$ is typically not spherical (that is, $|W|=\infty$ ). The building $(\Delta, \delta)$ is always thick, and it is locally finite and regular if and only if $\mathbb{F}=\mathbb{F}_{q}$ is a finite field, in which case $q_{s}=q$ for all $s \in S$.

An automorphism of $(\Delta, \delta)$ is a bijection $g: \Delta \rightarrow \Delta$ such that $\delta(g(x), g(y))=$ $\delta(x, y)$ for all $x, y \in \Delta$. We will call such automorphisms type preserving to distinguish from the slightly more general notion where diagram automorphisms of the underlying Coxeter system are permitted. A group $G$ of type preserving automorphisms is Weyl-transitive if for all chambers $x, y, x^{\prime}, y^{\prime} \in \Delta$ with $\delta(x, y)=$ $\delta\left(x^{\prime}, y^{\prime}\right)$ there exists $g \in G$ with $g . x=x^{\prime}$ and $g \cdot y=y^{\prime}$. Of course this implies that if $(\Delta, \delta)$ is locally finite then it is also regular. In Example 3.1 each $g \in G$ acts as a type preserving automorphism on $\Delta=G / B$ in the obvious way, and the group $G / K$ acts Weyl transitively (where $K$ is the kernel of the action).

Let $J \subseteq S$. Let $W_{J}=\langle\{s: s \in J\}\rangle$ be the standard parabolic subgroup of type $J$. The $J$-residue of a chamber $x \in \Delta$ is the set $R_{J}(x)=\left\{y \in \Delta: \delta(x, y) \in W_{J}\right\}$. It follows easily from the axioms that $\left(R_{J}(x),\left.\delta\right|_{R_{J}(x)}\right)$ is a building of type $\left(W_{J}, J\right)$. Let $\mathcal{R}$ be a residue and let $x \in \Delta$ be a chamber. There exists a unique chamber $y \in \mathcal{R}$ at minimal distance from $x$. This chamber is denoted $y=\operatorname{proj}_{\mathcal{R}}(x)$, and $\operatorname{proj}_{\mathcal{R}}: \Delta \rightarrow \mathcal{R}$ is called the projection onto $\mathcal{R}$. See [1, Section 5.3.2] for basic properties of projections.

Following [1, Chapter 12] there is a standard way to consider $(\Delta, \delta)$ as a simplicial complex by considering the partially ordered set of all spherical residues (that is, the $J$-residues with $\left.\left|W_{J}\right|<\infty\right)$. A simplex corresponding to a residue of type $J=\{s\}$ is called a panel (of cotype $s$ ). If $\Delta$ is locally finite then the associated simplicial complex is also locally finite (it is crucial here that only the spherical residues are considered), and thus its automorphism group totally disconnected, locally compact and also unimodular if it is Weyl-transitive ([3, Corollary 5]) .

The above simplicial complex has a natural geometric realisation $(X, d)$ as a CAT(0) space, called the Davis realisation of the building (see [1, Theorem 12.66]). Let us briefly describe some geometric properties of this space. Firstly, each apartment $\mathbb{A}$ of $X$ is a CAT $(0)$ realisation of the Coxeter complex of $(W, S)$. Thus there is a notion of walls in $\mathbb{A}$ as the fixed point sets of the reflections in $W$ (that is, the elements $w s w^{-1}$ with $w \in W$ and $s \in S$ ). Each wall divides $\mathbb{A}$ into exactly two connected components (called halfspaces), and a wall is said to separate points $x, y \in \mathbb{A}$ if $x$ and $y$ do not lie in a common halfspace. See, for example, [19] and [18] for further details. 
A wall in the Davis realisation $X$ is a wall in some apartment of $X$, and similarly a halfspace in $X$ is a halfspace in some apartment of $X$. We say that a wall in $X$ separates points $x, y \in X$ if this wall lies in some apartment containing $x$ and $y$ and separates these points in this apartment. Any geodesic in $X$ having a nondegenerate piece in a wall lies entirely in the wall, and halfspaces and walls are convex. Moreover, two points in $X$ are separated by a wall if and only if their carriers (defined as the simplex whose residue is the set of chambers whose realisation contains the point; see [1, Definition 12.19]), are separated by the wall. In particular, we have:

Lemma 3.2. The set of walls separating points in the Davis realisation equals the set of walls separating the supporting simplices of those points.

Recall that $\Delta$ is assumed to be locally finite, thick, and regular. If $\pi$ is a panel of cotype $s$, we define the thickness of $\pi$ to be $q_{\pi}=q_{s}$. Thus the residue of $\pi$ contains $q_{\pi}+1$ chambers. If $m$ is a wall of the Davis realisation, we define the thickness of $m$ to be $q(m)=q_{\pi}$ for any panel $\pi$ contained in $m$. This is well defined, because if $\pi$ and $\pi^{\prime}$ are panels contained in $m$ with associated residues $\mathcal{R}$ and $\mathcal{R}^{\prime}$ then it is easily seen that $\operatorname{proj}_{\mathcal{R}}: \mathcal{R}^{\prime} \rightarrow \mathcal{R}$ and $\operatorname{proj}_{\mathcal{R}^{\prime}}: \mathcal{R} \rightarrow \mathcal{R}^{\prime}$ are mutually inverse bijections. The thickness of a gallery $\gamma$ of type $s_{1}, \ldots, s_{n}$ is $q(\gamma)=q_{s_{1}} \cdots q_{s_{n}}$. Note that if $w=s_{1} \cdots s_{n}$ is a reduced expression then $q(\gamma)=q_{w}$. The thickness of a pair $x, y$ of simplices is defined to be

$$
q(x, y)=\prod_{m \in \mathcal{M}_{\mathbb{A}}(x, y)} q(m),
$$

where $\mathbb{A}$ is an apartment containing $x$ and $y$, and $\mathcal{M}_{\mathbb{A}}(x, y)$ denotes the set of walls of $\mathbb{A}$ separating $x$ and $y$. This value is independent of the particular apartment $\mathbb{A}$ containing $x$ and $y$ that we choose (this can be easily proven by considering the "convex hull" of the pair $x, y$, and noting that this convex hull is contained in every apartment that contains both $x$ and $y$ ). If $x$ and $y$ are singleton sets we write $q(x, y)$ instead of $q(\{x\},\{y\})$.

Every isometry of $X$ is semisimple by [6, Theorem A]. In other words, for every isometry, $g$ say, of $X$, the infimum in the following definition of the translation length of $g$, written $|g|$, is attained at some point of $X$.

$$
|g|:=\inf \{d(x, g \cdot x): x \in X\}
$$

The isometry induced by $g \in G$ is elliptic if and only if the scale of $g$ is 1 and is hyperbolic otherwise by [3, Theorem 7 and Corollary 10].

Definition 3.3. Let $g$ be an an isometry of $X$. The minimal set $\operatorname{Min}(g)$, of $g$ is the subset of $X$ defined by

$$
\operatorname{Min}(g):=\{x \in X: d(x, g \cdot x)=|g|\}
$$


The set $\operatorname{Min}(g)$ is closed, convex, and non-empty. The set $\operatorname{Min}(g)$ is the set of fixed points of $g$ if $g$ is elliptic and equals the union of the axes of $g$ if $g$ is hyperbolic.

\section{EXISTEnCE OF A SimpleX With TIDY STABILIZER}

For the computation of the scale of an element in $G$ we need a generalization of Proposition 4 in [3]. The following lemma is needed in its proof.

Lemma 4.1. Suppose that the action of a group $G$ on a building with $W$-distance $\delta$ is Weyl-transitive and type-preserving and let $a$ and $b$ be two simplexes whose residues are $\mathcal{A}$ and $\mathcal{B}$ respectively. Then

(1) the subgroup $G_{b}$ acts transitively on those simplices of the same type as a, whose residues $\mathcal{R}$ satisfy $\min (\delta(\mathcal{B}, \mathcal{R}))=\min (\delta(\mathcal{B}, \mathcal{A}))$

(2) the subgroup $G_{a} \cap G_{b}$ acts transitively on $\operatorname{proj}_{\mathcal{A}}(\mathcal{B})$, $\operatorname{proj}_{\mathcal{B}}(\mathcal{A})$ and the ordered pairs of chambers from these sets at Weyl-distance $\min (\delta(\mathcal{A}, \mathcal{B}))$.

Proof. We will use standard properties of projections between residues, compare for example section 5.3 in [1.

We first prove (1). Let $a^{\prime}$ be a simplex fitting the description given in the statement of our claim and let $\mathcal{A}^{\prime}$ be the corresponding residue. Denote by $c^{\prime}$ some chamber in $\operatorname{proj}_{\mathcal{B}}\left(\mathcal{A}^{\prime}\right)$ and let $d^{\prime}:=\operatorname{proj}_{\mathcal{A}^{\prime}}\left(c^{\prime}\right)$. Likewise choose a chamber, $c$ say, in $\operatorname{proj}_{\mathcal{B}}(\mathcal{A})$ and let $d:=\operatorname{proj}_{\mathcal{A}}(c)$. By our assumption on $a^{\prime}$ we have $\delta(c, d)=$ $\delta\left(c^{\prime}, d^{\prime}\right)=\min (\delta(\mathcal{B}, \mathcal{A}))$. By Weyl-transitivity, $(c, d)$ can be mapped to $\left(c^{\prime}, d^{\prime}\right)$ by some group element, which necessarily belongs to $G_{b}$, proving claim (1).

We next prove (2). Since the last statement clearly implies the others, we restrict ourselves to proving it. To that end, choose two ordered pairs of chambers in $\operatorname{proj}_{\mathcal{A}}(\mathcal{B}) \times \operatorname{proj}_{\mathcal{B}}(\mathcal{A})$. The Weyl-distance between the first and the second element of the pair is $\min (\delta(\mathcal{A}, \mathcal{B}))$ for both pairs. By Weyl-transitivity, the pairs can thus be mapped to each other by some group element, which necessarily belongs to $G_{a} \cap G_{b}$, proving the claim. The proof is complete.

Next we define a description of the relative position of two simplices with respect to the walls in the building which will play an important role in what follows.

Definition 4.2. Let $b$ and a be two (not necessarily distinct) simplices in a building with corresponding residues $\mathcal{B}$ and $\mathcal{A}$. We say

(1) the ordered pair $(b, a)$ is aligned if and only if $\operatorname{proj}_{\mathcal{B}}(\mathcal{A})=\mathcal{B}$.

(2) $a$ and $b$ are aligned if and only if $\operatorname{proj}_{\mathcal{B}}(\mathcal{A})=\mathcal{B}$ and $\operatorname{proj}_{\mathcal{A}}(\mathcal{B})=\mathcal{A}$.

The pair $(b, a)$ is aligned if and only if every wall that contains $b$ and belongs to an apartment that contains both $b$ and $a$ does also contain $a$. Therefore $a$ and $b$ are aligned if and only if every wall that belongs to an apartment that contains both $a$ and $b$ and contains either of these simplices also contains the other. 
The following proposition provides a way to measure the displacement between stabilizers of simplices with respect to $\mathbf{d}$ defined in Lemma 2.2. It will be used to provide a formula for the scale of isometries.

Proposition 4.3. Suppose that the action of a group $G$ on a building is Weyltransitive and type-preserving. Let $a$ and $b$ be two simplices with residues $\mathcal{A}$ respectively $\mathcal{B}$. Then

$$
\left|G_{b}: G_{b} \cap G_{a}\right|=\left|G_{b} \cdot \operatorname{proj}_{\mathcal{B}}(\mathcal{A})\right| \cdot q(b, a) .
$$

Proof. Using part (1) of Lemma 4.1 and the orbit-stabilizer theorem, we see that $\left|G_{b}: G_{b} \cap G_{a}\right|$ is equal to the number of simplices of the same type as $a$, whose residues $\mathcal{R}$ satisfy $\min (\delta(\mathcal{B}, \mathcal{R}))=\min (\delta(\mathcal{B}, \mathcal{A}))$. In order to count these, choose a fixed, reduced decomposition of $\min (\delta(\mathcal{B}, \mathcal{A}))$ and a set of chambers, $\mathcal{S}$ say, representing $G_{b} \cdot \operatorname{proj}_{\mathcal{B}}(\mathcal{A})$.

The residue of each cell in $G_{b} . a$ can be reached using a unique path leaving $\mathcal{B}$ following a gallery of the chosen type that starts with a chamber in $\mathcal{S}$. The number of these galleries equals $|\mathcal{S}| \cdot q(\gamma)$, where $\gamma$ is some gallery of the chosen type. Since the walls crossed by a gallery of the chosen type that ends in $\mathcal{A}$ are precisely those that separate $a$ from $b$, the statement claimed is verified.

\section{Remark 4.4.}

(1) By Lemma 4.1 $\mid G_{b}$. $\operatorname{proj}_{\mathcal{B}}(\mathcal{A})|=| \mathcal{B}|/| \operatorname{proj}_{\mathcal{B}}(\mathcal{A}) \mid$ if $(\Delta, \delta)$ is locally finite.

(2) The first factor on the right hand side of equation (2) in the conclusion of Proposition 4.3 simplifies to 1 if and only if the pair $(b, a)$ is aligned.

(3) An ordered pair beginning with a chamber is always aligned.

For a simplex whose stabilizer is tidy for an element, the mutual position of the simplex and its image under that element is special. As a first step to understanding the relationship of these simplices we note the following.

Lemma 4.5. Let $G$ be a closed, Weyl transitive subgroup of the group of type preserving automorphisms of a locally finite building. If the stabilizer of a simplex a is tidy for $g \in G$, then the pairs $\left(g^{n} \cdot a, g^{m} \cdot a\right)$ are aligned for all $n, m \in \mathbb{Z}$.

Proof. Since $G_{a}$ is tidy for $g$, it is also tidy for $g^{l}$ for each $l \in \mathbb{Z}$. Using Proposition 4.3 we have for fixed $l \in \mathbb{Z}$ and every $n \in \mathbb{N}$

(3) $k_{a, n l} \cdot q\left(g^{n l} \cdot a, a\right)=\left|G_{g^{n l} \cdot a}: G_{g^{n l} \cdot a} \cap G_{a}\right|=\left|G_{g^{l} \cdot a}: G_{g^{l} \cdot a} \cap G_{a}\right|^{n}=\left(k_{a, l} \cdot q\left(g^{l} \cdot a, a\right)\right)^{n}$

for some integers $k_{a, j}$ that are bounded above. Furthermore

$$
q\left(g^{n l} \cdot a, a\right) \leq\left(q\left(g^{l} \cdot a, a\right)\right)^{n} \quad \text { for all } n \in \mathbb{N}
$$

which implies

$$
k_{a, n l}=k_{a, l}^{n} \frac{\left(q\left(g^{l} \cdot a, a\right)\right)^{n}}{q\left(g^{n l} \cdot a, a\right)} \geq k_{a, l}^{n} \quad \text { for all } n \in \mathbb{N}
$$


Thus $k_{a, l}=1$ for all $l \in \mathbb{Z}$, which means that the pairs $\left(g^{l} \cdot a, a\right)$ are aligned for each integer $l$. Applying the automorphism $g^{n-l}$ we see that the pairs $\left(g^{n} \cdot a, g^{n-l} \cdot a\right)$ are aligned for each integer $l$. Putting $m:=n-l$ we obtain our claim.

Elliptic elements may fix vertices whose stabiliser is then a tidy subgroup for the element. This can not happen for hyperbolic elements.

Corollary 4.6. Let $G$ be a closed, Weyl-transitive subgroup of the group of typepreserving automorphisms of a locally finite building. Then the stabiliser of a vertex is not tidy for any hyperbolic element of $G$.

Proof. By Lemma 4.5 a simplex whose stabiliser is tidy for an element $g$ must form an aligned pair with its image under $g$. Since a pair of two vertices that are aligned are equal, any element that has the stabiliser of a vertex as a tidy subgroup must fix that vertex and hence is elliptic. The claim follows.

Our next result shows that we can always find a tidy subgroup for any element that equals the stabilizer of a simplex and compute the scale of the element from the walls separating the simplex and its image under the element in question.

Theorem 4.7. Let $G$ be a closed, Weyl transitive subgroup of the group of typepreserving automorphisms of a locally finite building. Let $g$ be an element of $G$, and $a$ be a simplex which is

(1) the carrier of some point in $\operatorname{Min}(g)$ if $g$ acts by elliptic isometries;

(2) the carrier of an open interval of an axis of $g$ no points of which are separated by a wall if $g$ acts by hyperbolic isometries.

Then $\left(g^{n} . a, a\right)$ is aligned for all $n \in \mathbb{Z}, G_{a}$ is tidy for $g$ and $s(g)=q(a, g . a)$.

Proof. Since the claims are obvious if $g$ acts by elliptic isometries, we may assume that $g$ acts by hyperbolic isometries in what follows.

We begin by verifying the claim on alignment. Observe that lemmata 4.2 and 4.1 in [9] imply that any wall in an apartment that contains a chosen axis of $g$ with the wall containing an open interval of that axis contains said axis completely. By the defining property of $a$, we conclude that $g^{n} . a$ and $a$ are aligned for any $n \in \mathbb{Z}$.

Applying Proposition 4.3 with $b=g^{n} . a$, part (2) of Remark 4.4 as well as Lemma 3.2 we conclude that for every $n \in \mathbb{N}$

$$
\left|g^{n} G_{a} g^{-n}: g^{n} G_{a} g^{-n} \cap G_{a}\right|=\left|G_{g^{n} . a}: G_{g^{n} . a} \cap G_{a}\right|=q\left(g^{n} . a, a\right) .
$$

The positioning of $a$ with respect to $\operatorname{Min}(g)$ implies

$$
\begin{gathered}
\forall n \in \mathbb{N}: q\left(a, g^{n} \cdot a\right)=q(a, g . a)^{n} \quad \text { which implies } \\
\forall n \in \mathbb{N}:\left|g^{n} G_{a} g^{-n}: g^{n} G_{a} g^{-n} \cap G_{a}\right|=\left|g G_{a} g^{-1}: g G_{a} g^{-1} \cap G_{a}\right|^{n},
\end{gathered}
$$

showing that $G_{a}$ is tidy for $g$ by Lemma 2.1. Since this implies that $s(g)=q(a, g . a)$, the proof is complete. 
The following theorem provides an alternative description of a simplex with tidy stabilizer that does not mention an axis for the element in question.

Theorem 4.8. Let $G$ be a closed group of Weyl-transitive, type-preserving automorphisms a thick, locally finite building. Let $g \in G$ and $a$ be a simplex that is aligned with g.a and such that $q(g . a, a)$ is minimal among all simplices with the same property. Then the stabilizer of a is tidy for $g$ and $s(g)=q(g . a, a)$.

Proof. This follows since tidy subgroups are minimizing and we already know that there is some simplex that is aligned with its image under $g$ whose stabilizer is a tidy subgroup for $g$.

Theorem 5.4 in [4] follows immediately from Theorem 4.8. We have therefore found a proof of that result that is both technically simple and valid in a much more general context.

\section{INTERPRETATION AS STRAIGHT DISPLACEMENTS}

The aim of this section is to characterize a simplex whose stabilizer is tidy for an element in an algebraic manner in terms of the Weyl group of the building, see Theorem 5.5. The apt term turns out to be the following.

Definition 5.1. Let $I \subseteq S$ be a spherical subset. An element $w \in W$ is called $I$-straight if $w$ is $(I, I)$-reduced, $w I w^{-1}=I$ and $\ell\left(w^{n}\right)=n \ell(w)$ for all $n \in \mathbb{N}$.

Since being straight is equivalent to being $\varnothing$-straight, $I$-straightness is a refinement of straightness. Furthermore, because $w I w^{-1}=I$ implies that conjugation by $w$ fixes $I$ pointwise, being $I$-straight implies being $J$-straight for each $J \subseteq I$.

We will require the following lemma. It is a special case of Theorem 2.1 in [2].

Lemma 5.2. Let $G$ be a closed group of Weyl-transitive, type-preserving automorphisms a thick, locally finite building. Let $I \subseteq S$ be spherical and let $P_{I}$ denote the stabilizer of the cotype I simplex of the base chamber. Then for all $(I, I)$-reduced elements $w \in W$ and $g \in G$ writing $Y(q)=\sum_{u \in Y} q_{u}$ for subsets $Y \subseteq W$ we have

$$
\left|\left\{h P_{I}: \delta\left(g P_{I}, h P_{I}\right)=w\right\}\right|=\frac{W_{I}(q)}{W_{I \cap w I w^{-1}}(q)} q_{w},
$$

Remark 5.3. The paper [2] assumes local finiteness and regularity of the building but no Weyl-transitive group action. Where all assumptions apply - as in our context - the following connection holds between the quotient $\frac{W_{I}(q)}{W_{I \cap w^{-1}(q)}}$ which appears in the general formula in Theorem 2.1 in [2] and the corresponding factor in Proposition 4.3. We may use part (1) of Remark 4.4 and see that the numerator of that quotient is the cardinality of the I-residue and the denominator is the cardinality of the projection of the J-residue at Weyl-distance $w$ on the I-residue.

The proposition below establishes the advertised characterisation of tidy subgroups for standard parabolics. The general case will be obtained by conjugating. 
Proposition 5.4. Let $G$ be a closed group of Weyl-transitive, type-preserving automorphisms a thick, locally finite building. Then the standard I-parabolic subgroup $P=P_{I}$ is tidy for $g \in G$ if and only if the element $w=\delta(P, g P)$ is I-straight.

Proof. ( $\Longrightarrow$ ) Suppose that $P$ is tidy for $g$. By Lemma 2.1 for all $n \in \mathbb{N}$ we have $\left|P: P \cap g^{-n} P g^{n}\right|=\left|P: g^{-1} P g\right|^{n}$, and it follows that $\left|P \backslash P g^{n} P\right|=|P \backslash P g P|^{n}$ for all $n \in \mathbb{N}$. Write $w_{n}=\delta\left(P, g^{n} . P\right)$ (so that $w_{n}$ is necessarily $(I, I)$-reduced, and $\left.w_{1}=w\right)$. We now argue similarly to the proof of Lemma 4.5. Lemma 5.2 gives for all integers $l$

$$
\frac{W_{I}(q)}{W_{I \cap w_{n l} I w_{n l}^{-1}(q)}} q_{w_{n l}}=\left(\frac{W_{I}(q)}{W_{I \cap w_{l} I w_{l}^{-1}(q)}}\right)^{n} q_{w_{l}}^{n},
$$

from which we deduce for all integers $l$ that

$$
\frac{W_{I}(q)}{W_{I \cap w_{n l} I w_{n l}^{-1}}(q)}=\left(\frac{W_{I}(q)}{W_{I \cap w_{l} I w_{l}^{-1}(q)}}\right)^{n} \frac{q_{w_{l}}^{n}}{q_{w_{n l}}} \geq\left(\frac{W_{I}(q)}{W_{I \cap w_{l} I w_{l}^{-1}}(q)}\right)^{n} .
$$

Since the left hand side is bounded above by $W_{I}(q)$, we deduce that $W_{I \cap w_{l} I w_{l}^{-1}}(q)=$ $W_{I}(q)$ for all $l$, and so $w_{l} I w_{l}^{-1}=I$ for all $l$. In particular, $w I w^{-1}=I$. Then formula (6) applied to $l=1$ gives $q_{w_{n}}=q_{w}^{n}$, for all integers $n$ and from this we deduce that $\ell\left(w_{n}\right)=n \ell(w)$ for all $n$. All that remains is to prove that $\ell\left(w^{n}\right)=$ $n \ell(w)$. We do this below by showing that in fact $w_{n}=w^{n}$ (and then use $\ell\left(w_{n}\right)=$ $n \ell(w))$.

Since $w_{n}=\delta\left(P, g^{n} P\right)$ there is a gallery of type $w_{n}$ joining a chamber of $P$ to a chamber of $g^{n} P$, and this gallery has minimal length amongst all such galleries. For each $i$, since $\delta\left(g^{i} P, g^{i+1} P\right)=\delta(P, g P)=w$ there is a reduced gallery of type $w$ joining a chamber of $g^{i} P$ to a chamber of $g^{i+1} P$. Connecting these galleries together using connecting galleries whose types are in $W_{I}$ produces a gallery of type

$$
w \cdot w_{1} \cdot w \cdot w_{2} \cdots w \cdot w_{n-1} \cdot w \quad \text { where } \quad w_{1}, \ldots, w_{n-1} \in W_{I},
$$

from a chamber of $P$ to a chamber of $g^{n} P$. Of course this gallery might not be of reduced type. However the part of type $w \cdot w_{1}$ is of reduced type (because $w$ is $(I, I)$-reduced). Since $w I w^{-1}=I$ we may write $w w_{1}=w_{1}^{\prime} w$ with $w_{1}^{\prime} \in W_{I}$, and it follows that there is also a reduced gallery of type $w_{1}^{\prime} \cdot w$ connecting the start and and chambers of the original gallery of type $w \cdot w_{1}$. So we make this distortion to produce a gallery of type

$$
w_{1}^{\prime} \cdot w \cdot w \cdot w_{2} \cdots w \cdot w_{n-1} \cdot w
$$

joining a chamber of $P$ to a chamber of $g^{n} P$. We can iterate this process to produce a gallery of type

$$
w_{1}^{\prime} \cdot w_{2}^{\prime} \cdots w_{n-1}^{\prime} \cdot \underbrace{w \cdot w \cdots w}_{n \text { terms }}
$$


joining a chamber of $P$ to a chamber of $g^{n} P$. The initial leg of the gallery of type $w_{1}^{\prime} \cdot w_{2}^{\prime} \cdots w_{n-1}^{\prime}$ stays completely inside the spherical parabolic $P_{I}$, and thus may be replaced by a gallery of type $w^{\prime} \in W_{I}$ of length bounded by $\operatorname{diam}\left(W_{I}\right)$. Thus we have produced a gallery of type

$$
w^{\prime} \cdot \underbrace{w \cdot w \cdots w}_{n \text { terms }}
$$

joining a chamber of $P$ to a chamber of $g^{n} P$.

Suppose that for some $n_{0} \in \mathbb{N}$ we have $\ell\left(w^{n_{0}}\right)<n_{0} \ell(w)$. It follows that the gallery of type $w \cdot w \cdots w$ ( $n_{0}$ terms) above can be replaced by a gallery of length bounded by $n_{0} \ell(w)-1$. Now consider the case $n=k n_{0}$. We have a gallery of type $w^{\prime} \cdot w \cdot w \cdots w$ (with $n$ factors $w$ ) from a chamber of $P$ to a chamber of $g^{n} P$. Break this gallery up as:

$$
w^{\prime} \cdot \underbrace{\underbrace{(w \cdots w)}_{n_{0} \text { terms }} \cdot \underbrace{(w \cdots w)}_{n_{0} \text { terms }} \cdots \underbrace{(w \cdots w)}_{n_{0} \text { terms }}}_{k \text { terms }} .
$$

Each of the $\underbrace{(w \cdots w)}_{n_{0} \text { terms }}$ parts of the gallery can be replaced by galleries of length at most $n_{0} \ell(w)-1$ connecting the same start and end galleries. Thus overall we produce a gallery of length at most

$$
\operatorname{diam}\left(W_{I}\right)+k\left(n_{0} \ell(w)-1\right)=n \ell(w)+\operatorname{diam}\left(W_{I}\right)-k=\ell\left(w_{n}\right)+\operatorname{diam}\left(W_{I}\right)-k .
$$

Thus choosing $k>\operatorname{diam}\left(W_{I}\right)$ we obtain a contradiction because we have a gallery of length strictly shorter than the minimum $\ell\left(w_{n}\right)$. Thus $\ell\left(w^{n}\right)=n \ell(w)$ for all $n \in \mathbb{N}$ and we are done.

$(\Longleftarrow)$ Suppose that $w=\delta(P, g P)$ is $(I, I)$-reduced, and that $w I w^{-1}=I$, and $\ell\left(w^{n}\right)=n \ell(w)$ for all $n \in \mathbb{N}$. Then

$$
P g^{n} P \subseteq P g P \cdot P g P \cdots P g P=P w P \cdot P w P \cdots P w P=P w^{n} P,
$$

and since double cosets are either disjoint or equal we have $P g^{n} P=P w^{n} P$. Therefore, using $w I w^{-1}=I$ and $\ell\left(w^{n}\right)=n \ell(w)$ we have

$$
\left|P \backslash P g^{n} P\right|=\left|P \backslash P w^{n} P\right|=\frac{W_{I}(q)}{W_{I \cap w^{n} I w^{-n}}(q)} q_{w^{n}}=q_{w}^{n}=|P \backslash P w P|^{n}=|P \backslash P g P|^{n} .
$$

Thus $\left|P: P \cap g^{-n} P g^{n}\right|=\left|P: g^{-1} P g\right|^{n}$ for all $n \in \mathbb{N}$ and so $P$ is tidy for $g$ by Lemma 2.1.

We now deduce the announced tidiness criterion for stabilizers of simplices in terms of Weyl-displacement in general.

Theorem 5.5. Let $G$ be a closed group of Weyl-transitive, type-preserving automorphisms a thick, locally finite building and let $g \in G$. Then the stabilizer of a simplex $a$ is tidy for $g$ if and only if $\delta(a, g . a)$ is cotype(a)-straight. 
Proof. The simplex $a$ is of the form $h P$ with $P$ a standard parabolic of cotype $(a)$.

By Propositon 5.4,

$$
\delta(a, g . a)=\delta(h P, g . h P)=\delta\left(P, h^{-1} g h . P\right)
$$

is cotype $(a)$-straight if and only if $P$ is tidy for $h^{-1} g h$. But this is equivalent to $h P h^{-1}$, the stabilizer of $h P=a$ being tidy for $g$. The proof is complete.

Definition 5.6. For a type preserving automorphism $g$ of a building $\Delta$ we call any straight element in $\{\delta(a, g . a): a$ is a simplex of $\Delta\}$ a straight displacement of $g$.

Theorem 5.5 has implication for the values of the scale function.

Corollary 5.7. Let $G$ be a closed group of Weyl-transitive, type-preserving automorphisms a thick, locally finite building. Then the scale of any element $g$ of $G$ is the q-value of a straight displacement of $g$ (possibly trivial).

Proof. Let $g \in G$. Choose a simplex $a$ in $\Delta$ whose stabilizer is tidy for $g$; this is possible by Theorem 4.7. By the same Theorem, we have $s(g)=q(a, g . a)$, which equals $q_{\delta(a, g . a)}$. The element $\delta(a, g . a)$ is cotype $(a)$-straight by Theorem 5.5 , in particular it is straight. This proves our claim.

We will improved upon this corollary in Corollary 7.3 .

\section{Geometric Characterisation of Simplices With tidy Stabilizer}

We now establish, for a hyperbolic element, a connection between metric and combinatorial axes in the reverse direction to Theorem 4.7. In a special case this result is part of the content of Lemma 5.4 in [10].

Theorem 6.1. Let $G$ be a closed group of Weyl-transitive, type-preserving automorphisms a thick, locally finite building. Let a be a simplex whose stabiliser is tidy for a hyperbolic element $g \in G$. Then there is an axis of $g$ that intersects the interior of the geometric realisation of a.

Proof. We may suppose that $a$ is a face of the fundamental chamber, $c_{0}$. Then $w:=\delta(a, g . a)$ is cotype $(a)$-straight. Hence, by Lemma 3.2 in [19 the element $w$ has an axis, $L$ say, in the Davis-realisation of the Coxeter complex of $W$ that is contained in all walls of $c_{0}$ of type $i$ for all $i \in \operatorname{cotype}(a)$ and hence passes through an interior point of $a$.

It follows that the set $g^{\mathbb{N}} \cdot c_{0}$ is $W$-isometric to a subset of an apartment, hence contained in an apartment. Therefore the geometric realization of this apartment contains the image of $L$ which is an axis of $g$ that intersects $a$ and contains an interior point of it.

A direct, geometric construction of an axis without recourse to a straight displacement of the isometry is of interest.

In terms of straight displacements we can reformulate the theorem as follows. 
Corollary 6.2. Let $G$ be a closed group of Weyl-transitive, type-preserving automorphisms a thick, locally finite building. Then every element $g$ in $G$ attains a straight Weyl-displacement $\delta(a, g . a)$ only for simpices a whose relative interior intersects $\operatorname{Min}(g)$.

Proof. Theorem 5.5 implies that the stabilizer of the simplex $a$ is tidy for $g$. If $g$ is elliptic, $\delta(a, g \cdot a)=1$, the simplex $a$ is fixed by $g$ and the claim holds. If $g$ is hyperbolic, then there is an axis of $g$ that intersects the relative interior of $a$ by Theorem 6.1.

Remark 6.3. It is interesting to what extent straight displacements of a group element $g$ are unique. Only the case of hyperbolic $g$ is in question, since the unique Weyl-displacement of elliptic elements is the trivial element. For hyperbolic $g$ it can be shown that all such displacements on simplices that contain interior points of an axis of $g$ are conjugate. As straight elements of Coxeter groups satisfy very tight restrictions, it is conceivable that stricter restrictions apply.

We can conclude that minimal displacement of points in the Davis-realisation is more or less the same as minimal displacement of their stabiliziers.

Corollary 6.4. Let $G$ be a locally compact group that acts Weyl-transitively by type-preserving automorphisms of a thick building, and let $g \in G$. Then for a point $x$ in a Davis-realisation of the building the distance between $G_{x}$ and $G_{g . x}$ is minimal if and only if $x$ does not lie on a wall that separates the attracting and repelling points of $g$ at infinity and there is a point $x^{\prime}$ in the carrier of $x$ such that the distance in the Davis-realisation between $x^{\prime}$ and $g . x^{\prime}$ is minimal.

Proof. The condition on the walls eliminates precisely the points lying on essential walls for $g$.

For any point $x$, the distance between $G_{x}$ and $G_{g . x}$ equals the distance between $G_{a}$ and $G_{g . a}$, where $a$ is the carrier of $x$. If this distance is minimal among all distances between $G_{y}$ and $G_{g . y}$ it must equal the minimal distance between a compact, open subgroup of $G$ and its conjugate under $g$ by Theorem 4.7 (that is, the distance is equal to $\left.\log (s(g))+\log \left(s\left(g^{-1}\right)\right)=2 \log (s(g))\right)$, and $G_{x}$ is tidy for $g$.

Theorem 6.1 then shows that the carrier of $x$ contains another point $x^{\prime}$ which lies on an axis for $g$ as claimed.

The reverse direction is similar, but easier, and is left to the reader.

This corollary has an interesting consequence, which will be elaborated on in more detail in Section 7, compare Remark 7.2.

Remark 6.5. Notice that the distance between points according to the CAT(0)metric is roughly proportional to the number of walls the geodesic connecting these points crosses, while the distance between the stabilizers of those points is equal to the sum of the logarithms of the thicknesses of the walls the geodesic crosses. This corollary is therefore somewhat surprising, since, in the case when the building has 
walls with different thicknesses, it appears to say that (type-preserving) hyperbolic automorphisms will pass through walls in a way which utilises the different available thicknesses in a balanced manner.

The formulation of Corollary 6.4 would be much neater if (metric) minimal sets of group elements would always be subcomplexes. However, this is not the case as is illustrated by the following example.

Example 6.6. Pictured below is a detail of the Davis-realisation (with standard choice of distances to walls) of an apartment with Coxeter group $\mathrm{PGL}_{2}(\mathbb{Z})$ together with an illustration of the minimal set of a hyperbolic isometry (between the dotted gray lines with a fundamental domain under powers of the hyperbolic isometry filled in gray) together with the geometric realisation of a chamber and its image under the isometry (pictured in blue and green respectively).

The walls are shown in black. You can also see the hexagons and squares used in the dual complex to represent the corresponding spherical residues. The Davisrealisation shown arises as the universal cover of the 4-6-12-tessalation of the plane with 12-gons removed subdivided by walls as indicated in the detail. The hyperbolic element is a lift of a translation symmetry of that tessalation.

You can find a picture of this Davis-complex overlaid over the Poincaré model of the hyperbolic plane as figure 12.11 on page 625 in [1]. That version shows an overview over the whole complex, but it is not metrically accurate. From that picture however, it is clear that the Davis-complex is a thickening of the Bass-Serre tree of $\mathrm{PGL}_{2}(\mathbb{Z})$.

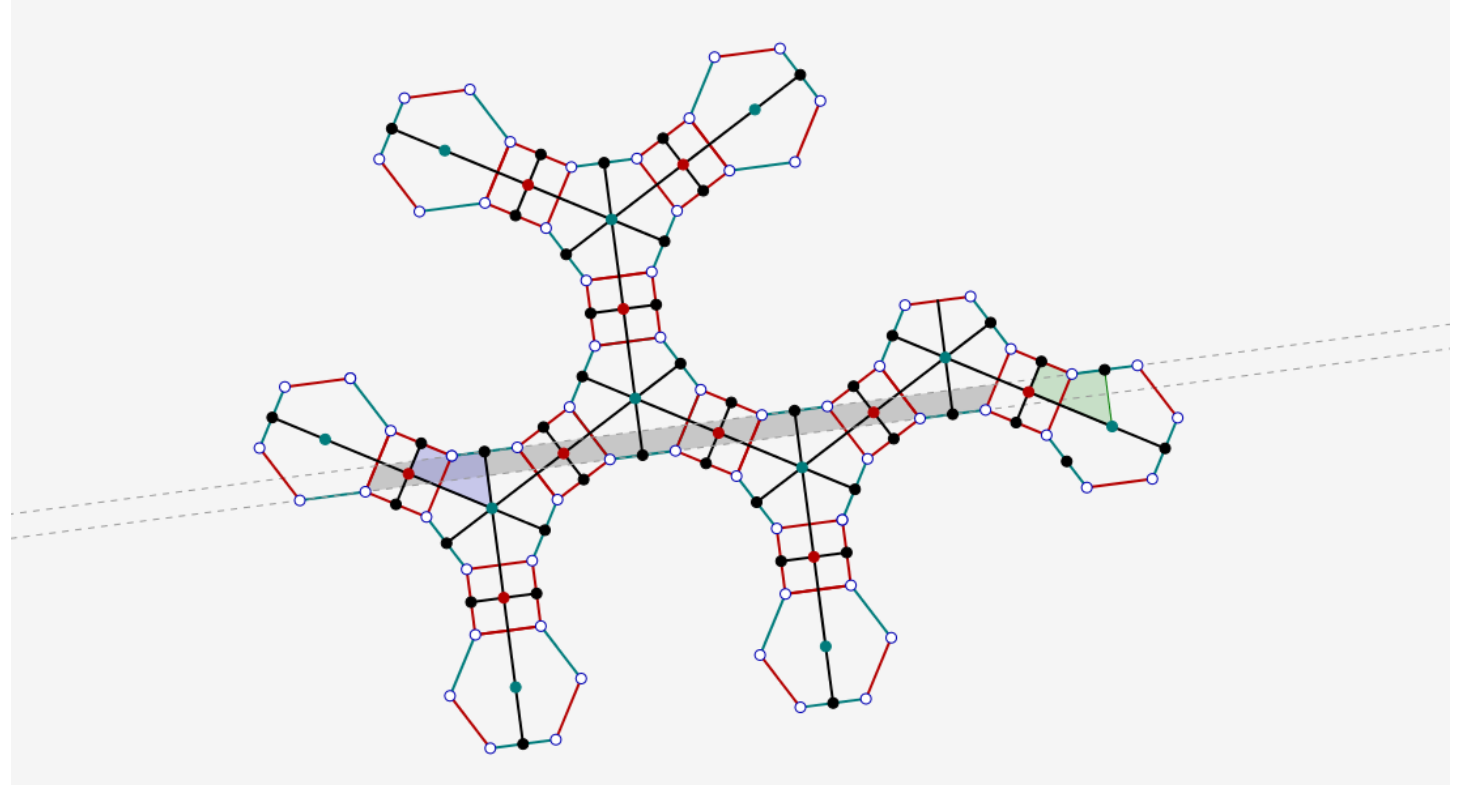

It is seen here that the minimal set of the chosen translation is not a subcomplex. Indeed, an even more extreme trait can be seen in the same example by choosing the distances to the walls that factor in the construction of the Davis-realisation 
differently. If we increase the distance of the vertices of the quadrangles from the walls that do not intersect the hexagons sufficiently, the minimal set of the "same" isometry degenerates into a single geodesic.

While this example lives within a thin building, the example can be thickened in a manner that is equivariant with respect to the hyperbolic isometry illustrated.

The following figure makes it easier to verify some of the claims made above. It shows a detail of the underlying 4-6-12-tessalation with walls extended into the 12-gons, which in the universal cover widen into a horoball-neighborhood of the cut-out vertices at infinity seen in the Poincaré-model.

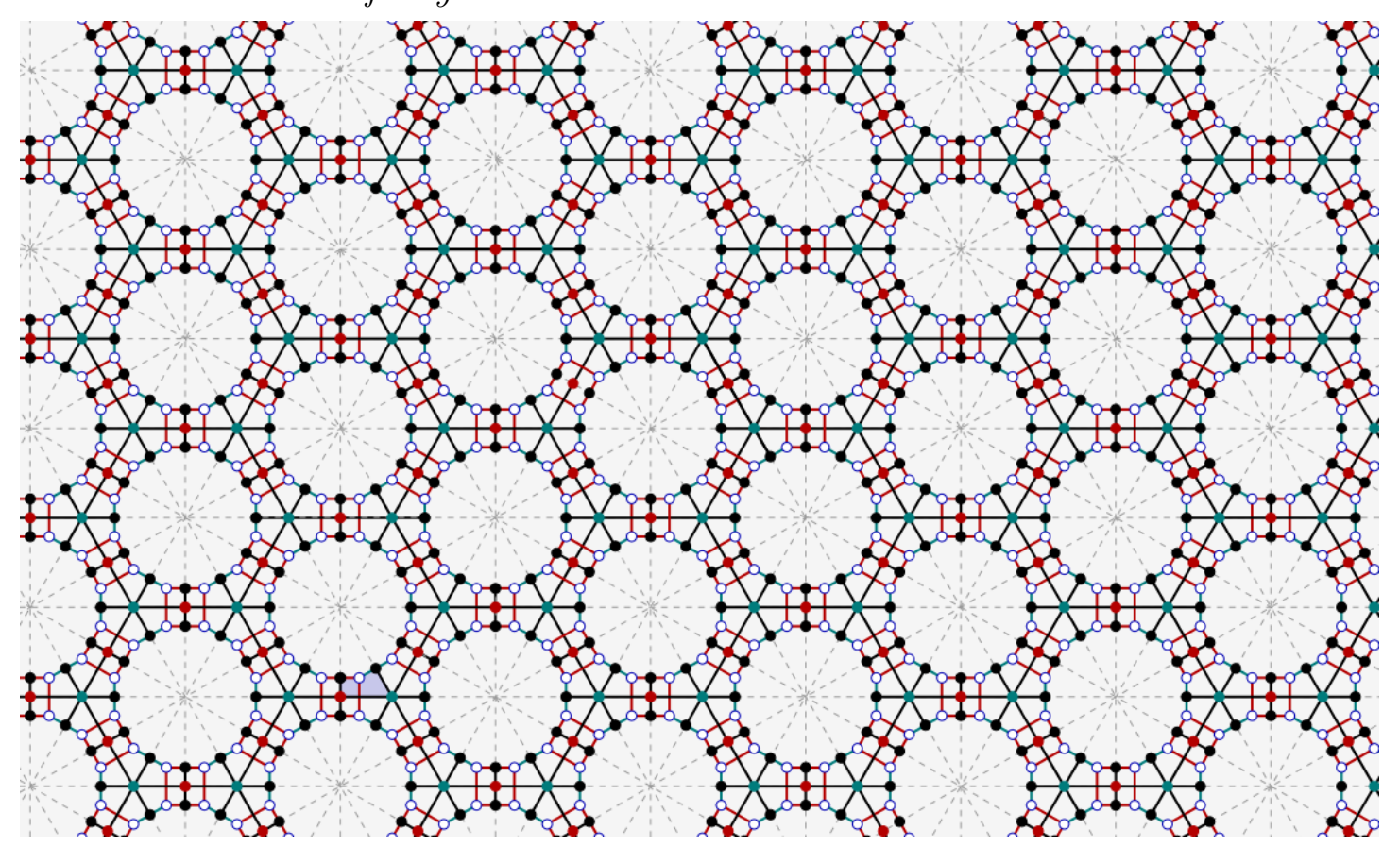

\section{Applications}

Proposition 7.1. Let $G$ be a closed group of Weyl-transitive, type-preserving automorphisms a thick, locally finite building of type $(W, S)$. Let I be a subset of $S$ and $w \neq 1$ an $I$-straight element of $W$. Further, let $a$ and $b$ be simplices of cotype $I$ and Weyl-distance $w$. Then there exists a hyperbolic element $g$ of $G$ such that some axis of $g$ contains interior points of $a$ and $b$ and $g . a=b$.

Proof. Choose a simplex $x \neq b$ of cotype $I$ at Weyl-distance $w$ from $a$. This is possible, since the number of elements of Weyl-distance $w$ from $a$ is at least $q_{w} \geq 2$.

Choose chambers $c_{x}, c_{a}$ and $c_{b}$ such that $x$ is a face of $c_{x}, a$ is a face of $c_{a}$ and $b$ is a face of $c_{b}$ and $\delta\left(c_{x}, c_{a}\right)=\delta\left(c_{a}, c_{b}\right)=w$. Then, by Weyl-transitivity of the action of $G$, pick $g \in G$ such that $g \cdot c_{x}=c_{a}$ and $g . c_{a}=c_{b}$.

By construction, and because $g$ preserves types, we have $g . a=b$ and $\delta(a, g \cdot a)=$ $\delta(a, b)=w$. By Theorem $5.5 G_{a}$ is tidy for $g$. The simplices $a$ and $b$ are therefore 
aligned by Lemma 4.5 and we may compute the scale of $g$ using Proposition 4.3 to be $q(a, b)=q_{w}>1$. We conclude that $g$ is hyperbolic. We obtain the claim about the position of an axis of $g$ from Theorem 6.1. The proof is complete.

Remark 7.2. We can now make Remark6.5 more precise. Since by the above Proposition, every non-trivial straight Weyl-displacement can be realised with the action of some hyperbolic element on an axis, Corollary 6.4 appears to imply that there is a restricion on the thicknesses of different types of walls purely in terms of the Weyl group, limited only by the straight elements contained therein.

Note that straight elements are widespread in infinite Coxeter groups by Theorems 4.1.5 and 4.1.6 in [18].

Another interesting consequence of Proposition 7.1 is the following corollary, which improves on Corollary 5.7.

Corollary 7.3. Let $G$ be a closed group of Weyl-transitive, type-preserving automorphisms a thick, locally finite building of type $(W, S)$. Then the set of scale values of $G$ equals $\left\{q_{w}: w\right.$ is a straight element of $\left.W\right\}$. In particular, $G$ is uniscalar if and only if $W$, and hence $\Delta$, are finite; or put differently, if and only if $\Delta$ is spherical.

Proof. The scale of any element of $G$ is the $q$-value of some straight element of the Weyl group by Corollary 5.7. Proposition 7.1 then shows, that all the scale values listed in the statement above are in fact realised. This shows the first statement.

To derive the last statement, note that $G$ is uniscalar if and only if all of its elements are elliptic. The latter necessarily happens if $\Delta$ is finite. If on the other hand, $\Delta$ is infinite, then its Weyl group is infinite as well. Now every infinite Coxeter group contains non-trivial straight elements; any Coxeter element in an infinite, irreducible component will do, see [15, Corollary F]. The remaining claim follows form this and the part of our claim that we already proved.

The important class of rank one isometries can also be described by their straight displacements as shown in the next result.

Proposition 7.4. Let $G$ be a closed group of Weyl-transitive, type-preserving automorphisms a thick, locally finite building $(\Delta, \delta)$ of type $(W, S)$. Then a hyperbolic element $g \in G$ is not a rank one isometry of the Davis-realisation of $(\Delta, \delta)$ if and only if some, equivalently every, straight displacement of $g$ is contained in $W_{I} \times W_{J}$, where either $W_{I}$ and $W_{J}$ are both infinite, or $W_{I}$ is affine and $W_{J}$ is finite.

Proof. Let $w$ be a straight displacement of $g$ and $L$ an axis of $g$ that passes through a simplex $a$ whose Weyl-displacment $\delta(a, g . a)$ equals $w$. Further, let $A$ be an apartment that contains $L$; it will then also contain $a$. Choose a chamber $c$ of $A$ that contains $a$ and let $\rho$ be the retraction onto $A$ centered at $c$.

Then $\rho \circ g$ restricts to an automorphism of $A$ which is given by $w$ if $A$ is identified with the Coxeter complex of $W$ by mapping $c \in A$ to $1 \in W$. 
Assume that $w$ is contained in $W_{I} \times W_{J}$, where either $W_{I}$ and $W_{J}$ are both infinite, or $W_{I}$ is affine and $W_{J}$ is finite. Then we conclude that $w$ stabilizes $W_{I} \times W_{J}$, and thus - varying the apartment $A$ - the isometry $g$ stabilizes a residue of type $W_{I} \times W_{J}$ in $\Delta$. By Theorem 5.1 in [8] $g$ is not a rank one isometry.

If, conversely, $g$ is not a rank one isometry, the arguments of the implication $($ iii $) \Rightarrow(i)$ in the proof of Theorem 5.1 in [8] show that every straight displacement of $g$ is contained in $W_{I} \times W_{J}$, where either $W_{I}$ and $W_{J}$ are both infinite, or $W_{I}$ is affine and $W_{J}$ is finite. The proof is complete.

Furthermore, we suspect that Weyl-displacements of arbitrary simplices under a hyperbolic isometry $g$ can be computed from the position of the simplex relative to an axis of $g$ and a suitable straight displacement of $g$ on this axis.

\section{REFERENCES}

[1] Peter Abramenko and Kenneth S. Brown. Buildings, volume 248 of Graduate Texts in Mathematics. Springer, New York, 2008. Theory and applications.

[2] Peter Abramenko, James Parkinson, and Hendrik Van Maldeghem. Distance regularity in buildings and structure constants in Hecke algebras. J. Algebra, 481:158-187, 2017.

[3] Udo Baumgartner, Bertrand Rémy, and George A. Willis. Flat rank of automorphism groups of buildings. Transform. Groups, 12(3):413-436, 2007.

[4] Udo Baumgartner, Günter Schlichting, and George A. Willis. Geometric characterization of flat groups of automorphisms. Groups Geom. Dyn., 4:1-13, 2010.

[5] Udo Baumgartner and George A. Willis. The direction of an automorphism of a totally disconnected locally compact group. Math. Z., 252:393-428, 2006.

[6] Martin R. Bridson. On the semisimplicity of polyhedral isometries. Proceedings of the American Mathematical Society, 127(7):2143-2146, 1999.

[7] Martin Bridson and André Haeflinger. Metric Spaces of non-positive Curvature, volume 319 of Grundlehren der mathematischen Wissenschaften. Springer Verlag, 1999.

[8] Pierre-Emmanuel Caprace and Koji Fujiwara. Rank-one isometries of buildings and quasimorphisms of Kac-Moody groups. Geom. Funct. Anal., 19(5):1296-1319, 2010.

[9] Pierre-Emmanuel Caprace and Frédéric Haglund. On geometrical flats in the CAT(0) realization of Coxeter groups and Tits buildings. Canad. J. Math., 61:740-761, 2009.

[10] Pierre-Emmanuel Caprace and David Hume. Orthogonal forms of Kac-Moody groups are acylindrically hyperbolic. Ann. Inst. Fourier (Grenoble), 65(6):2613-2640, 2015.

[11] S.G. Dani and N. Shah and G.A. Willis. Locally compact groups with dense orbits under $\mathbb{Z}^{d}$-actions by automorphisms. Ergodic Theory \& Dynam. Systems, 26:1443-1465, 2006.

[12] H. Glöckner. Scale functions on p-adic Lie groups. Manuscripta Math. 97:205-215, 1998.

[13] N. Iwahori and H. Matsumoto. On some Bruhat decomposition and the structure of the Hecke rings of p-adic Chevalley groups. Publ. Math., Inst. Hautes Étud. Sci., 25:5-48, 1965.

[14] W. Jaworski and J.M. Rosenblatt and G.A. Willis. Concentration functions in locally compact groups. Math. Ann., 305:673?691, 1996.

[15] Timothée Marquis. Conjugacy classes and straight elements in Coxeter groups. J. Algebra, 407:68-80, 2014.

[16] Cheryl Praeger, Jacqui Ramagge and George A. Willis. A graph-theoretic description of the scale-multiplicative semigroups of automorphisms Preprint, available at https://arxiv.org/pdf/1710.00439.pdf. 
[17] Rögnvaldur G. Möller. Structure theory of totally disconnected locally compact groups via graphs and permutations. Canadian Journal of Mathematics, 54:795-827, 2002.

[18] G.A. Noskov. Asymptotic behavior of word metrics on Coxeter groups. Doc. Math., J. DMV, 16:373-398, 2011.

[19] Guennadi A. Noskov and Èrnest B. Vinberg. Strong Tits alternative for subgroups of Coxeter groups. J. Lie Theory, 12(1):259-264, 2002.

[20] J. Parkinson. Buildings and Hecke algebras. J. Algebra, 297:1-49, 2006.

[21] Y. Shalom and G.A. Willis. Commensurated subgroups of arithmetic groups, totally disconnected groups and adelic rigidity. Geometric and Functional Analysis, 23:1631-1683, 2013.

[22] J. Tits. Buildings of spherical type and finite $B N$-pairs. Lecture notes in Mathematics, Springer-Verlag, 368, 1974.

School of Mathematics and Statistics, University of Sydney, Sydney NSW 2006, Australia Tel.: +61-2 93514221 FAx: +61-2 93514534

E-mail address: Udo.Baumgartner@sydney.edu.au

School of Mathematics and Statistics, University of Sydney, Sydney NSW 2006, Australia Tel.: +61-2 93514221 Fax: +61-2 93514534

E-mail address: James.Parkinson@sydney.edu.au

School of Mathematics and Statistics, University of Sydney, Sydney NSW 2006 , Australia Tel.: +61-2 93514533 FAX: +61 293514534

E-mail address: Jacqui. Ramagge@sydney.edu .au 